\title{
Importance of interparticle gaps for tailoring the properties of composites
}

\author{
G.Bossis, C.Métayer,S.Lacis,C.Abbo \\ LPMC,UMR 6622, University of Nice,Parc Valrose,06108 Nice Cedex 2 \\ TEL:(33)4 92076775 FAX: :(33)492076754 E-mail bossis@unice.fr \\ (Received 26, July 2000 Accepted 30, November 2000)
}

\begin{abstract}
Electroactive elastomers are composites made of solid particles embedded in an elastomeric network whose electrical, mechanical or optical properties can be changed by the application of an electric or a magnetic field. These materials have obviously a strong connection with ER and MR fluids and can be more appropriated for some applications. We present results concerning the conductivity, magnetic permeability and elasticity of an elastomer filled with iron or carbonyl iron particles. We show that the change of elastic properties obtained by the application of a magnetic field depends strongly on the gap between the particles. We explain quantitatively the experimental results with the help of finite element calculation to predict the magnetic forces between the particles. The effect of a magnetic field on the electrical resistance is also investigated and we show that we can obtain a huge decrease of the resistance by the application of a magnetic field.
\end{abstract}

Keywords: adaptative materials, magnetic elastomers, magnetoresistance

\section{I.INTRODUCTION}

Magnetorheological and Electrorheological fluids are suspensions of micronic particles which both suffer from the gravity: the sedimentation is always present and can lead to the irreversible aggregation of the particles. Some trick can be used in order to prevent sedimentation like the addition of compounds that form a physical gel with a low yield stress. Nevertheless we have found that the presence of a silica gel which prevents the sedimentation can significantly decrease the yield stress ${ }^{1}$ and so the performances of the suspension as a controlable fluid. At the opposite, if the polarizable particles are embedded into an elastomeric matrix, then the sedimentation problem is of course ruled out but the question is now about the utility of such a compound. Solid particles (for instance carbon black or silica ) are often used in rubber industry as fillers to reinforce the mechanical properties of the elastomers. Now, if the fillers react to an external imposed field, then we have got a smart material whose intrinsic properties can be controlled by external means. For instance the stiffness of an elastomer filled with magnetic particles can be controlled by the application of a magnetic field. This possibility has been demonstrated several years ago by different teams $\mathrm{s}^{2,3,4}$ and some applications for the active control of vibrations are being developped. We can also envisage the use of electro-active elastomers but the need of high electric field imposes the use of sheets of thicknesses smaller than one millimeter which reduces their range of application for the control of vibrations. The adaptative characteristics of these materials are mainly due to the fact that some material properties (like magnetic permeability or electrical conductivity ) are very sensitive to the structure and especially to the gap between particles. It is this aspect that we are going to develop in the following section.

\section{SAMPLES}

The elastomer matrix was made from a bicomponent silicone purchased from Rhône-Poulenc under the name RTV (room temperature vulcanizing)581. The particles are made either of iron (average diameter 50 microns) or of carbonyl iron with an average diameter of 2 microns. Two kinds of samples were realized. In the first one, that we shall call isotropic, the iron particles are mechanically dispersed in the prepolymer and the blend is poured into a cylindrical mould before curing takes place. In the second kind of sample called "structured" we have applied a magnetic field during the curing process in order to align the magnetic particles in the direction of the field. We are going to compare the Young modulus and the electrical conductivity of these two kinds of samples for different volume fractions and also as a function of an applied external field. 


\section{EFFECT OF A MAGNETIC FIELD ON THE ELASTICITY}

In our experiments the samples are cylinders of length $50 \mathrm{~mm}$ and diameter $7 \mathrm{~mm}$. This sample is placed inside the core of a coil whose length is 250 $\mathrm{mm}$ and internal core $30 \mathrm{~mm}$. An inner cyndrical shell allows to cool the coil with a circulation of water and to keep constant the temperature during the experiment. The stress increase due to the application of different magnetic fields is shown in Fig.1 as a function of the strain. These curves correspond to the sample where the polymerization has been done after the particles have been aligned (anisotropic sample); for the unstructured sample the stress increase is an order of magnitude lower. It is interesting to note that at zero strain, the fact to turn on the magnetic field gives a stress of about $20 \mathrm{kPa}$ for the highest field. This stress, which does not exist for isotropic samples, comes from the attraction between almost touching particles. These particles have been aggregated by the application of a magnetic field during the curing process but one can imagine that it remains many gaps between the particles and that those gaps are responsible for the force at zero strain. Actually this zero strain magnetic force is an important part of the response of the elastomer to the magnetic field. For $\mathrm{H}=97 \mathrm{kA} / \mathrm{m}$ we have distinguished in the Figure 1 the zero strain contribution $(14 \mathrm{kPa})$ from a stretching component which is maximum $(7 \mathrm{kPa})$ for a strain of $4 \%$. The strain corresponding to a maximum of the extra stress shifts from zero for the lowest field to $5 \%$ for the maximum field we have applied $(123 \mathrm{kA} / \mathrm{m})$. Actually this distinction between the zero strain stress and a stretching stress is quite arbitrary since when the elastomer extends, the initial gaps between the particles will increase and their

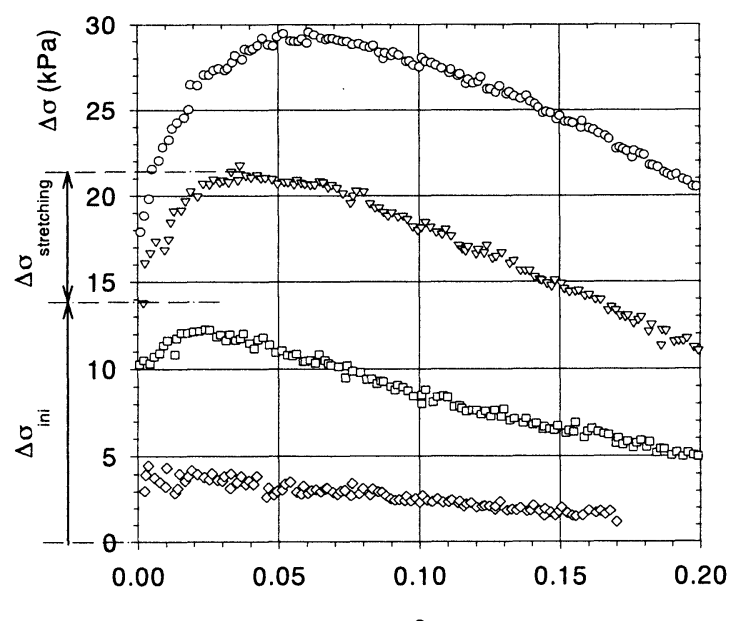

Fig.1 : Stress due to the magnetic field

From top to bottom :

$\mathrm{H}=123 \mathrm{kA} / \mathrm{m}, 97 \mathrm{kA} / \mathrm{m}, 65 \mathrm{kA} / \mathrm{m}, 32 \mathrm{kA} / \mathrm{m}$.

Volume fraction : $\Phi=30 \%$ contribution to the total magnetic force will decrease instead of remaining constant. We can try to explain the observed behavior by saying that, on one hand we have a magnetic stress which comes from the existence of small gaps between particles, and on the other hand we have a reinforced elastic stress which originates from the presence of chains of particles which do not break as long as the imposed elastic stress is lower than the magnetic stress between two particles in contact. These chains reinforce the composite as fibers do: by enhancing the local elastic stress. This mechanism allows to understand why we observe that the maximum effect is obtained at a strain which increases with the amplitude of the magnetic field: this is because the particles begin to separate (or the network to slip) only when the elastic stress is higher than the magnetic stress. We can compare the increase of stress due to the application of the field to the one which can be calculated from the force between two spheres. We use a finite element calculation which has been improved in order to properly deal with the large change of magnetic energy in the vicinity of the contacts between particles ${ }^{5}$. The geometry is similar to the one used by Ginder et $\mathrm{al}^{6}$ : we keep the cylindrical symmetry and the unit cell includes two half particles separated by a distance $2 \varepsilon$ a with periodic boundary conditions along the symmetry axis. The size of the unit cell is calculated from the volume fraction and the magnetic field tangent to the edge of the cubic cell is the average magnetic field. The force between the two spheres is obtained from an integral of the magnetic stress tensor on a plane perpendicular to the symmetry axis and located in the gap between the two spheres. The magnetization for the carbonyl iron is represented by the Frohlish-Kennely law: $\mu=1+\frac{M_{s}\left(\mu_{\text {ini }}-1\right)}{M_{s}+\left(\mu_{\text {ini }}-1\right) H}$ with the saturation magnetization; $\quad M s=1990 \mathrm{kA} / \mathrm{m}$ and initial permeability : $\mu_{\text {ini }}=132$. The resulting stress as a function of the strain is shown in Fig.2. The solid line is the theoretical result for a field $\mathrm{H}=123 \mathrm{kA} / \mathrm{m}$. The closed symbols are the experimental results. We can note that, contrarily to the experimental value, the theoretical stress decreases strongly with the strain, furthermore its initial value is almost five times larger than the experimental one. The explanation of this discrepancy is likely the following: in the model the local strain is the average strain and is represented by the same gap between each particle; in practice the particles stay at contact and when the stress is increased the polymeric network slips on the surface of the particles. The extra stress is then given by a yield stress which corresponds to the beginning of a relative motion between the particles and the network. This yield stress corresponds to the different maxima 


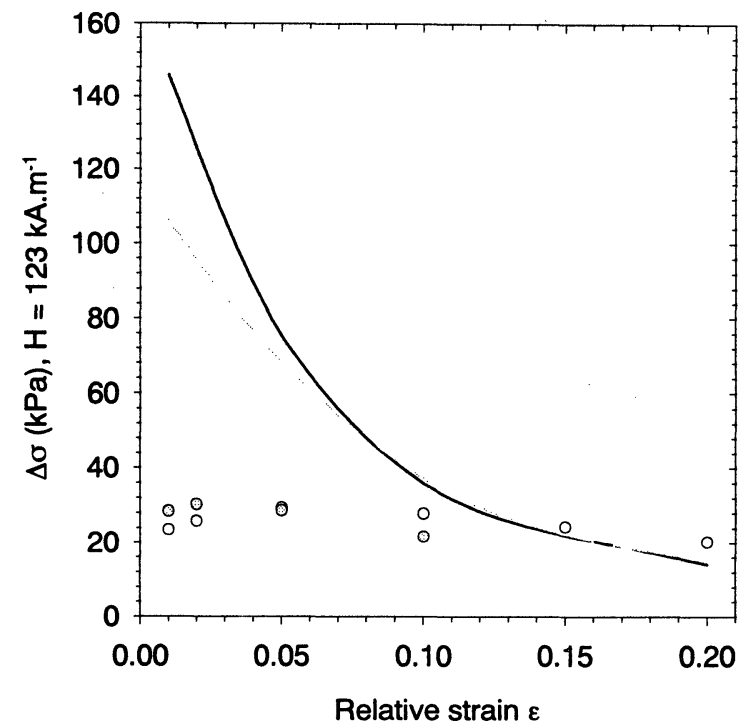

Fig. 2 Comparison theory-experiment

- : FEM calculation for chains of spheres 00000 : Experimental values for $\Phi=25 \%$

presented in Figure 1; above this yield stress we would expect that the extra stress would not change as long as the applied stress remains lower than the one which is supposed to break the contacts between the particles (more than $160 \mathrm{kPa}$ for $\mathrm{H}=123 \mathrm{kA} / \mathrm{m}$ ). This is not entirely true since we observe a slight decrease, but we have to remember that initially not all the particles are in contact since we have an initial stress at zero strain. Then the gaps between these initially separated particles will continue to increase under the increasing applied stress and will reduce the contribution of the extra magnetic stress. These are the combination of these two effects (slipping on particles at contact and increasing gap between particles initially separated which can explain the observed experimental data.

\section{CHANGE OF RESISTIVITY INDUCED BY A MAGNETIC FIELD}

In the preceding section we have seen that when the particles are aligned before curing, the elasticity of the composite can be changed a lot by applying a magnetic field. The key point is the formation of a continuous path of the particulate phase which is broken when the material is strained. If the contrast between the properties of the matrix and the one of the particles is important, then a creation of gaps between the particles will have a large effect on the macroscopic transport properties and especially on the resistivity. Beside possible applications many theoretical questions are arising in order to explain the measurement of the electrical conductivity of an electrostructured composite with conductive filler particles. Firstly the conduction of the composite is very dependent on the quality of the contacts between the particles. These contacts are altered by the presence of oxide layers and also by the roughness of the surfaces. The resistivity of a metallic powder can be decreased tremendously with the help of an electromagnetic radiation. This effect was discovered by Branly in 1890 and was used in the first wireless telegraph receiver. A similar effect may occur if the powder is submitted to a DC voltage : above a threshold voltage which was $28 \mathrm{~V}$ in our experiment (cf Fig3), the powder becomes conductive and remains so even if the voltage is removed. The conductive state can be destroyed by a small mechanical shock but also a pressure can change considerably and reversibly the resistance of the powder. It has been shown by thermal imaging that the conducting paths are the one where the relative pressure between grains is maximum ${ }^{7}$. We have observed the Branly effect both on isotropic and structured materials. The results obtained in the isotropic state are shown in Fig. 4. The upper line is the resistance of the sample (a cylindrical sheet of thickness $2 \mathrm{~mm}$ and and radius $1 \mathrm{~cm}$ ) in the non conductive state as a function of the volume fraction of the iron particles, whereas the lower one is the resistance in the conductive state (applied voltage $50 \mathrm{~V})$. We see clearly the existence of a percolation threshold at a volume fraction of about $25 \%$ which appears both in the conductive and not conductive states, but what is surprising is the existence of a conductive state for volume fractions well below this percolation threshold. On the contrary, for the sample which has been structured before polymerisation, we only observe a continuous decrease of the resistance when we increase the volume fraction from $5 \%$ to $30 \%$ and the final resistance is only slightly lower than for the isotropic sample.

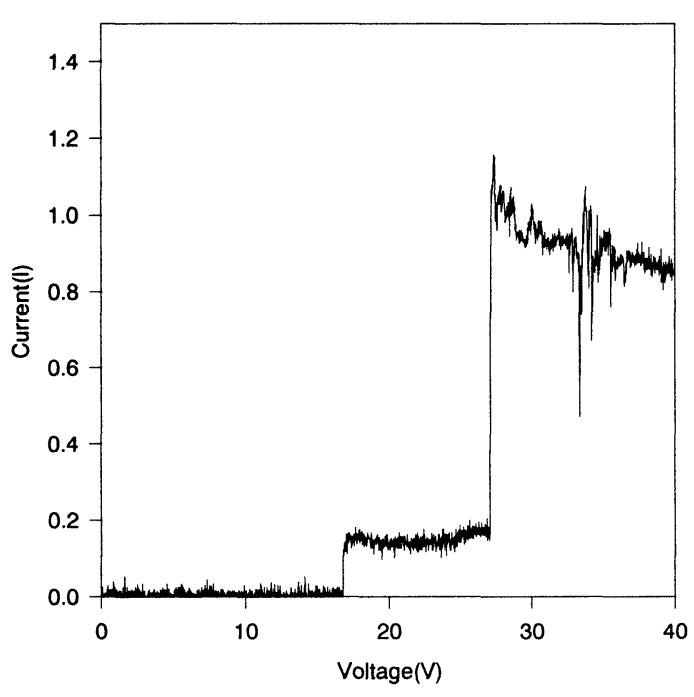

Fig.3 Transition towards a conducting state by increasing the applied voltage 


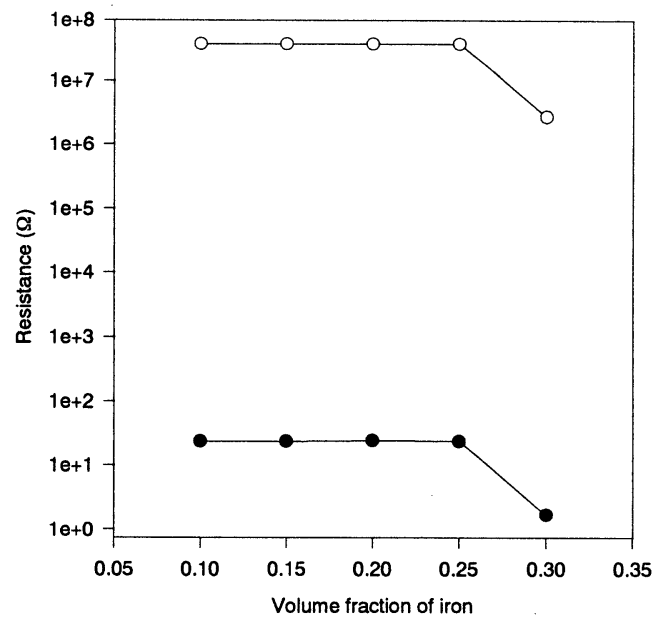

Fig.4 Resistance as a function of the volume fraction of iron for an isotropic state of filler particles;

upper curve: insulating state;

lower curve: conducting state

The most interesting feature is perhaps the change of resistivity induced by the application of a magnetic field. This effect is only obtained on a structured material and is due to the magnetostrictive pressure which increases the quality of the contacts between the particles. The change of resistivity versus the applied magnetic field is shown in Fig. 6 and this is obtained in the insulating state. We see that increasing the field from zero to 6000 Oersted decreases the resistivity by five orders of magnitude. This is a huge effect. When the field is decreased we have an important hysteresis loop. This is likely due to the fact that the magnetic pressure has allowed the

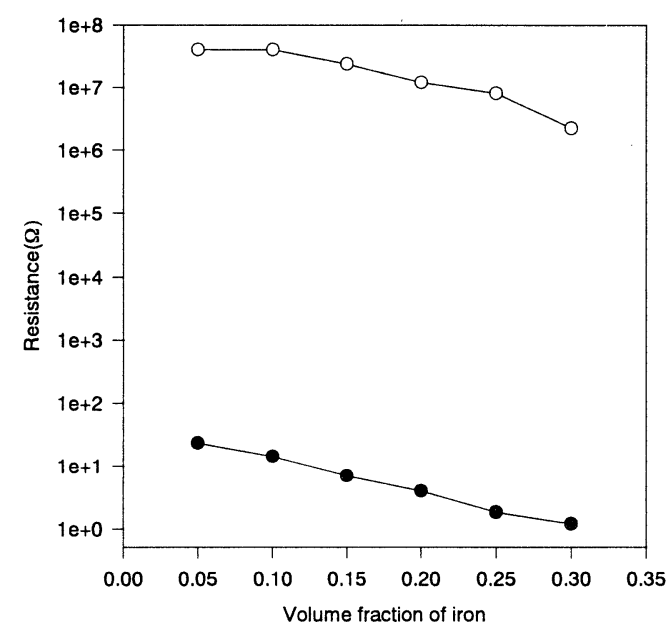

Fig.5 Resistance as a function of the volume fraction of iron after alignment of filler particles; upper curve: insulating state;

lower curve: conducting state formation of solid microcontacts between the particles. When the magnetic pressure is released the solid micro-contacts do not break immediately but only when the elastic recovering force between the particles becomes larger than the mechanical strength of these micro-contacts. The resistivity appears to be much more sensitive to the gaps between the particles than the magnetic permeability and should be useful to test the relative motion of particles when the composite is strained under a magnetic field. It is also worth noting that the curve presented in Fig.6 has been obtained for a sample which is cured inside a rigid box; it means that in the presence of the magnetic field there is no possibility for an overall magnetostriction of the sample. The change of resistivity is then only due to tiny rearrangements between the particles caused by the local magnetic forces.

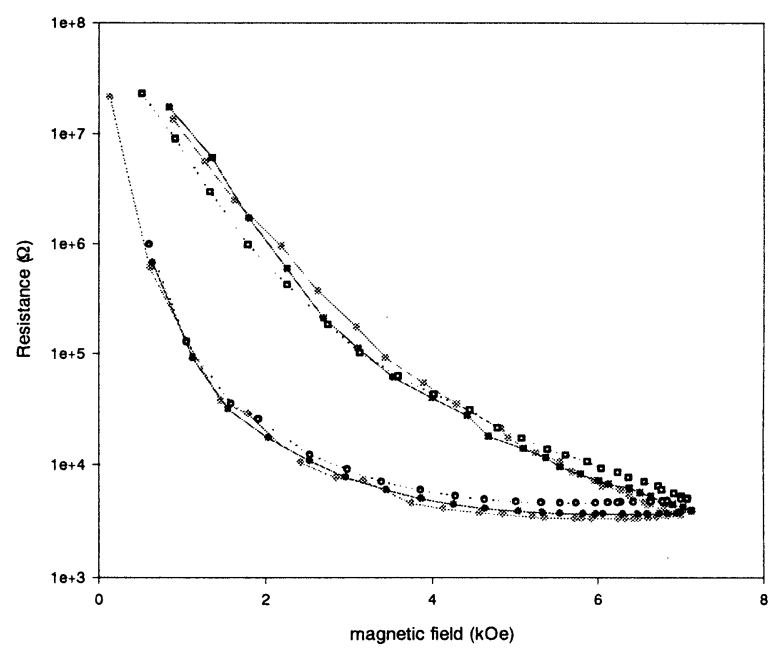

Fig.6 Change of resistance with the applied magnetic field;

upper curves: increasing field

lower curves: decreasing field

\section{CONCLUSION}

In this paper we have presented experimental results which show how much the macroscopic properties of a composite made of magnetic particles inside an elastomeric matrix can be sensitive to the interparticle distance between particles. This is true for the change of elasticity in the presence of a magnetic field and this is still better emphasized by the change of conductivity in the presence of a magnetic field. Besides the applications which can be found for these materials, it is also useful to be able to get some informations about the relative motion of the particles when the material is submitted to an 
oscillatory strain. A combined measurement of electrical and mechanical properties would certainly helps a lot for improving the understanding of the mechanical response of filled elastomers.

\section{REFERENCES}

1. O.Volkova.PhD thesis (in English), University of Nice,October 1998

2. G.Bossis, E.Lemaire,. SFP Colloquium, 4iéme journées de la matière condensée-Rennes 31 August-2 September 1994

3. M.R.Jolly,J.D.Carlson, B.C.Munoz, T.A.Bullions. Jof Intelligent . Materials. Systems and Structures.7 (1996) 613-622.
4. Patent number 04266970- A Toyota Cent Res \& Dev Lab (in Japanese)

5. S.Lacis,O.Volkova,G.Bossis Proceedings of "Modelling of material Processing",Riga,May 28-29,1999,pp222-227

6. J.M.. Ginder, L.C.. Davis, LD., Elie, Proceedings of the 5Th International Conference on ER fluids, W.A.Bullough, ed. Word Scientific, Singapore, 1996, pp. 504-514

7. D.Vandembroucq,A.C.Boccara,S.Roux J.Phys. III France 7(1997) 303-310

Presented at 3rd Japan France Seminar on IMS 